\title{
MODELAGEM MATEMÁTICA DE UM GIROSCÓPIO SINTONIZADO DINAMICAMENTE
}

\author{
Ettore Apolônio de Barros \\ EPUSP - ESCOLA POLITÉCNICA DA UNIVERSIDADE DE SÃO PAULO \\ Departamento de Engenharia Mecatrônica e Sistemas Mecânicos \\ Av Prof Mello Moraes, 2231, Cidade Universitária, CEP 05508-900 - São Paulo, SP. \\ Fernando de Castro Junqueira \\ CTMSP - CENTRO TECNOLÓGICO DA MARINHA EM SÃO PAULO \\ Av Prof Lineu Prestes, 2468, Cidade Universitária, CEP 05508-000 - São Paulo, SP.
}

\begin{abstract}
This work describes the principle of operation and the motion dynamics of a dynamically tuned gyroscope, DTG. The complete set of equations of motion are derived, and a simplified representation for the transfer functions are obtained, emphasizing the concepts related to important parameters, and phenomena that affect the DTG performance.
\end{abstract}

KEYWORDS: Gyroscope, inertial navigation system, sensor, DTG.

\section{RESUMO}

Este trabalho apresenta a modelagem matemática da dinâmica de operação de um giroscópio sintonizado dinamicamente, DTG. A obtenção das funções de transferência simplificada ocorre realçando-se os conceitos relacionados a parâmetros e fenômenos importantes no desempenho do sensor.

PALAVRAS CHAVES: Giroscópio, sistema de navegação inercial, sensor, DTG.

\section{INTRODUÇÃO}

Giroscópios sintonizados dinamicamente, DTG, têm sido desenvolvidos desde 1945, como uma alternativa de baixo custo aos giroscópios eletro-mecânicos convencionais,

Artigo Submetido em 03/08/04

1a. Revisão em 23/11/04;

2a. Revisão em 30/03/05;

Aceito sob recomendação do Editor Associado

Prof. Dr. Takashi Yoneyama que utilizam um rotor imerso em óleo com temperatura controlada. Pode-se chegar a reduções de até 75\% no custo sem grandes comprometimentos na precisão (derivas de 0,01 grau/hora são geralmente atingidas). A tecnologia envolvida no seu projeto e fabricação também não é sofisticada. Estes fatores o tornam atraente como opção de desenvolvimento a baixo custo e de rápidos resultados em comparação com o desenvolvimento de outros tipos de sensores inerciais. Tais características também estimularam o desenvolvimento e estudo destes sensores no Centro Tecnológico da Marinha em São Paulo (Junqueira, 2003; Junqueira e Barros , 2003) e no Instituto de Pesquisas Espaciais(Garrotti, 2003) .

Para tratar-se do seu princípio de funcionamento, os elementos básicos deste tipo de sensor estão ilustrados em Figs. 1 e 3, onde um rotor de inércia é ligado ao eixo de rotação através de uma suspensão do tipo Cardan, denominada aqui de "cruzeta", composta por um anel interno e barras de torção.

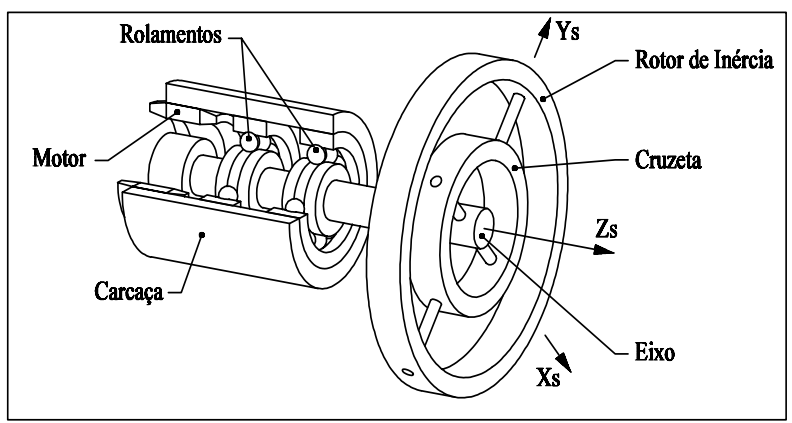

Figura 1 - Representação esquemática de um DTG 
A reação inercial da cruzeta a um deslocamento angular do rotor em relação à carcaça é relacionada ao fenômeno do binário giroscópico, e varia quadraticamente com a velocidade de rotação do eixo. Por outro lado, a rigidez constante das barras de torção é responsável por um torque aplicado no sentido contrário ao esforço anterior. Assim sendo, conforme ilustrado na Fig. 2., para um determinado valor de rotação, denominado de rotação de sintonia, ocorre o cancelamento de ambos os esforços, o que faz, teoricamente, com que o rotor gire livremente no espaço.

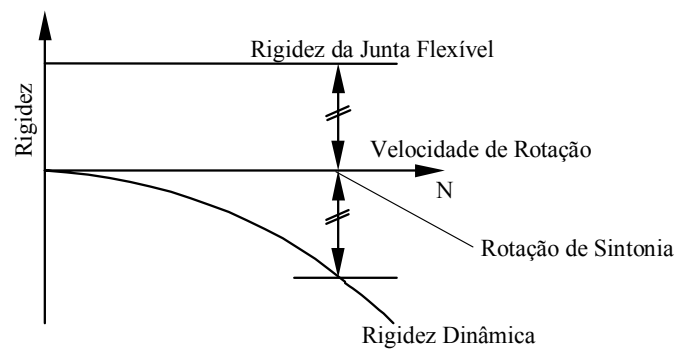

Figura 2- Princípio de Funcionamento do DTG

Para que o rotor acompanhe os deslocamentos angulares da carcaça, bobinas de torque aplicam momentos no rotor de forma a causar sua precessão, cuja intensidade varia com o deslocamento do rotor em relação à carcaça, medido por sensores eletromagnéticos de proximidade (Fig. 3). Os torques aplicados são proporcionais às correntes nas bobinas, que funcionam também como valores do deslocamento angular absoluto da carcaça medido pelo sensor.

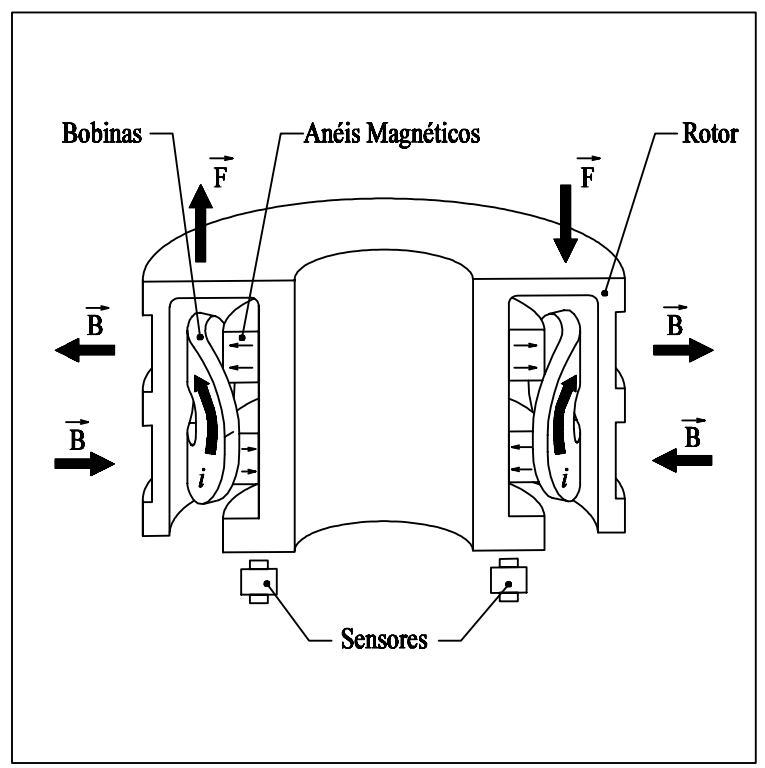

Figura 3- Posicionamento das bobinas de torque e sensores de proximidade no sistema de controle de um DTG.
O pleno entendimento da resposta dinâmica de um DTG passa pela análise do seu modelo matemático, que envolve razoável complexidade. Os artigos de Craig (1972a, 1972b), tomados na literatura como referência principal no que se refere à modelagem matemática do DTG, apresentam linguagem pouco afeita a modelos de controle, além de passagens e simplificações de pouca clareza, bem como pequenos erros, que serão apontados neste trabalho. Outras referências, como Lawrence (1998) e IEEE (1989), apresentam nível insuficiente de profundidade.

$\mathrm{Na}$ derivação das equações de movimento do DTG, Garrotti (2003) segue com maior fidelidade os passos desenvolvidos em Craig(1972 a), adotando a notação complexa para a descrição conjunta dos movimentos de rotação em dois eixos ortogonais. Além disso, como em Craig(1972 a), trata de um caso mais genérico , já que constantes de rigidez e momentos de inércia relativos a eixos transversais não são admitidos de mesmo valor, bem como o número de anéis da cruzeta pode ser arbitrário. Modelos lineares são derivados e comparados com aqueles apresentados em IEEE(1989). Nessa referência, inclui-se também a modelagem e análise da influência dos componentes eletrônicos na malha de controle do DTG, o que não é escopo deste e dos trabalhos de Craig.

Este trabalho também se insere num contexto de esclarecimento e discussão dos resultados apresentados originalmente por Craig. Ao contrário de Garroti(2003), no entanto, deriva-se as funções de transferência do DTG em termos de conceitos físicos palpáveis e observáveis na prática, tais como os movimentos de precessão e nutação, bem como se enfatiza o papel de parâmetros importantes na avaliação do sensor, nomeadamente a figura de mérito e as constantes de amortecimento.

$\mathrm{O}$ equacionamento , a ser apresentado, é feito para um caso particular: uma cruzeta, bem como juntas elásticas idênticas. Não se utiliza a notação complexa, e apresentam-se explicitamente as funções de transferência entre movimento da carcaça e do rotor. Acredita-se que essa abordagem proporcione uma maior facilidade no entendimento inicial da dinâmica do DTG , e que seja de mais fácil tratamento na análise e projeto do seu sistema de controle. Procura-se também integrar o modelo de Craig aos resultados mais práticos apresentados nas normas de teste de DTGs proposto pelo IEEE (1989).

Nas hipóteses simplificadoras que são utilizadas, supõe-se um DTG típico ,de bom desempenho, com parâmetros cujos valores são exemplificados na tabela 1 . 
Tabela 1 - Parâmetros típicos de um DTG.

\begin{tabular}{|l|l|}
\hline 1 - PARÂMETRO & 2 - VALOR \\
\hline Velocidade Angular do eixo motor & $118 \mathrm{~Hz}$ \\
\hline Velocidade Angular de Nutação & $200 \mathrm{~Hz}$ \\
\hline Constante de Tempo de Precessão & $100 \mathrm{~s}$ \\
\hline Constante de Tempo de Nutação & $100 \mathrm{~s}$ \\
\hline Desvio da Condição de Sintonia & $5 \mathrm{~Hz}$ \\
\hline Figura de Mérito & 500 \\
\hline
\end{tabular}

\section{RELAÇÕES CINEMÁTICAS}

\subsection{Movimento do Eixo e sua Relação com o Movimento da Carcaça}

No desenvolvimento a seguir, supõe-se que os deslocamentos angulares tratados sejam de pequena magnitude (a folga entre rotor e carcaça permite, em geral, deslocamentos máximos de 0,5 grau). Desta forma, a hipótese de deslocamentos angulares infinitesimais, que podem ser tratados vetorialmente, será adotada.

Sejam os sistemas de coordenadas solidários ao eixo $\left(x_{s}, y_{s}, z_{s}\right)$, à cruzeta $\left(x_{g}, y_{g}, z_{g}\right)$, ao rotor $\left(x_{r}, y_{r}, z_{r}\right)$ e à carcaça $\left(x_{c}, y_{c}, z_{c}\right)$, conforme as figuras 4 a 6 . Os eixos $y$ (na direção das barras internas) e $z$ do eixo e cruzeta coincidem, bem como os eixos $x$ do rotor $\mathrm{e}$ cruzeta. Seja $\overrightarrow{\dot{\phi}}$, o vetor velocidade angular da carcaça em relação ao referencial inercial. Este possui componentes $\dot{\phi}_{x c}$ e $\dot{\phi}_{y c}$ no sistema fixo à carcaça, que são as entradas do sistema dinâmico considerado. O eixo move-se com velocidade angular de módulo $\mathrm{N}$ constante, em relação à carcaça. Logo, as componentes do vetor velocidade do eixo, expressadas no sistema solidário ao mesmo (Fig.5), são:

$$
\begin{aligned}
& \omega_{x s}=\dot{\phi}_{x c} \cdot \cos N t+\dot{\phi}_{y c} \cdot \operatorname{sen} N t \\
& \omega_{y s}=-\dot{\phi}_{x c} \cdot \operatorname{sen} N t+\dot{\phi}_{y c} \cdot \cos N t \\
& \omega_{z s}=N
\end{aligned}
$$

\subsection{Movimento do Rotor}

Este será expresso pelas parcelas que representam o movimento do eixo somado ao movimento do rotor em relação ao eixo. As componentes do vetor velocidade

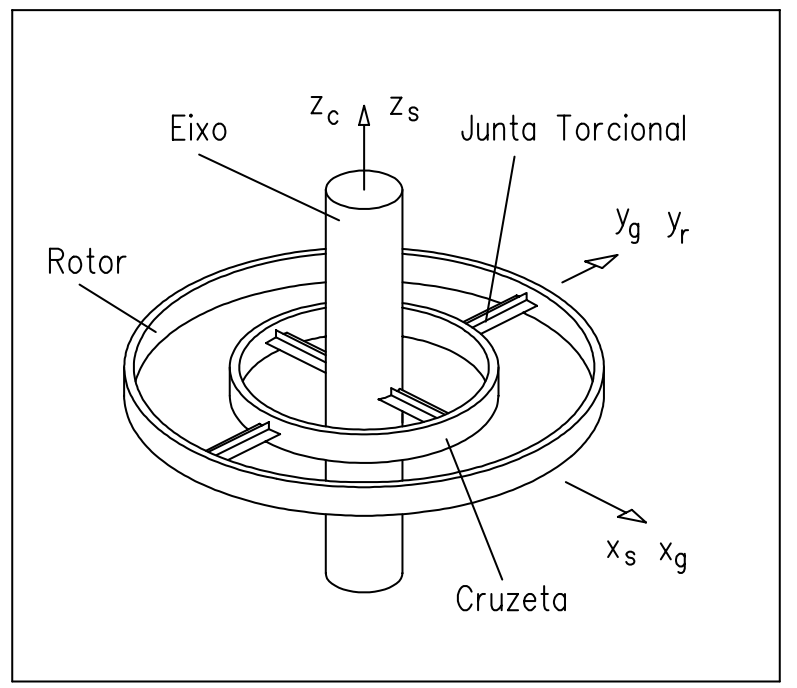

Figura 4 - Eixo, Rotor (anel externo), Cruzeta (anel interno e juntas) e os respectivos sistemas de coordenadas solidários.

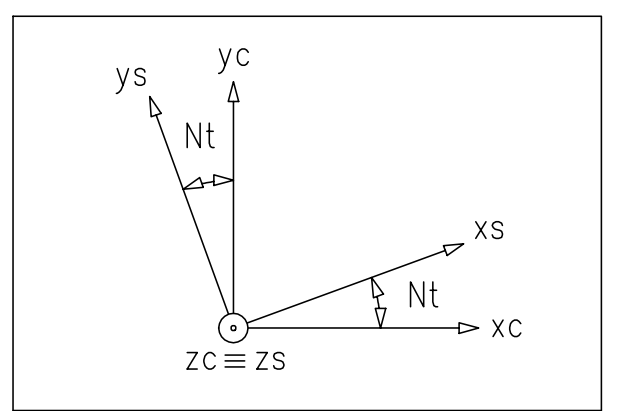

Figura 5 - Transformação da Carcaça para o Eixo

angular em relação à carcaça serão representadas no sistema solidário ao rotor (Fig. 6):

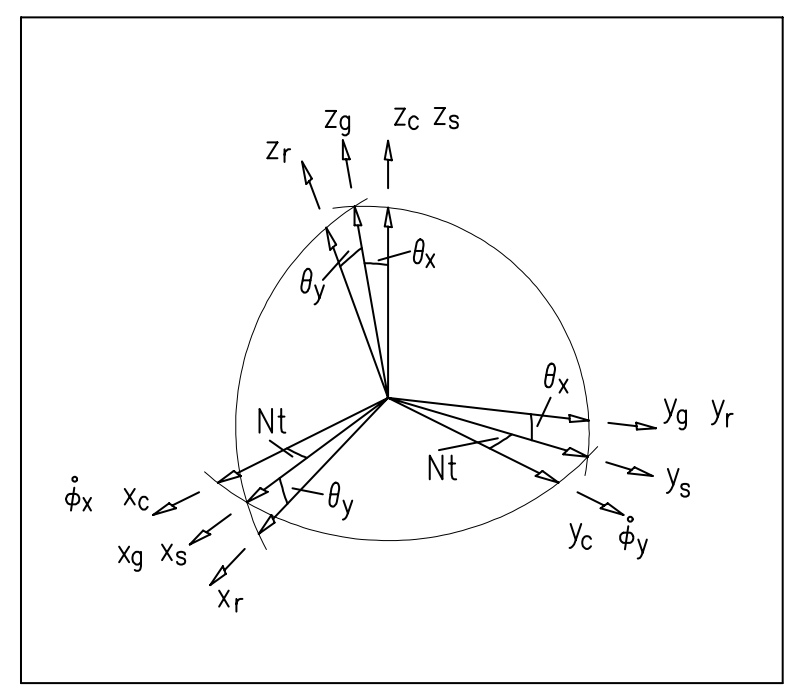

Figura 6-Deslocamento angular do rotor. 


$$
\begin{aligned}
& \omega_{x r}=\omega_{x s}-N \cdot \theta_{y}+\dot{\theta}_{x} \\
& \omega_{y r}=\omega_{y s}+N \cdot \theta_{x}+\dot{\theta}_{y} \\
& \omega_{z r}=\omega_{x s} \cdot \theta_{y}-\omega_{y s} \cdot \theta_{x}+N \cong N
\end{aligned}
$$

Em relação ao referencial fixo na carcaça, o deslocamento angular do rotor é expresso por:

$$
\begin{aligned}
& \theta_{x c}=\theta_{x} \cos N t-\theta_{y} \operatorname{sen} N t \\
& \theta_{y c}=\theta_{x} \operatorname{sen} N t+\theta_{y} \cos N t
\end{aligned}
$$

Ou seja,

$$
\begin{gathered}
\theta_{x}=\theta_{x c} \cos N t+\theta_{y c} \operatorname{sen} N t \\
\theta_{y}=-\theta_{x c} \operatorname{sen} N t+\theta_{y c} \cos N t
\end{gathered}
$$

\section{3- Movimento da Cruzeta}

A junta é solidária à rotação do rotor em torno do eixo $x_{g}$, mas é independente de rotações do rotor em torno de $y_{g}$. As componentes de sua velocidade angular em relação à carcaça, no sistema solidário à cruzeta, são:

$$
\begin{aligned}
& \omega_{x g}=\omega_{x s}+\dot{\theta}_{x} \\
& \omega_{y g}=\omega_{y s}+N \cdot \theta_{x} \\
& \omega_{z g}=-\omega_{y s} \cdot \theta_{x}+N \cong N
\end{aligned}
$$

\section{EQUAÇÕES DINÂMICAS}

Aplicaremos, a seguir, as equações de Euler aos elementos cruzeta e rotor para exprimir a variação de seus respectivos momentos angulares em função dos torques externos aplicados.

\section{1- Esforços na Cruzeta}

O momento resultante na cruzeta resulta da diferença entre os torques aplicados pelo rotor e eixo. Este é igualado à variação do seu momento angular expresso no sistema solidário à cruzeta, pelo seu centro de massa. Ou seja:

$$
\begin{aligned}
& \sum M_{x g}=A_{g} \cdot \dot{\omega}_{x g}+\left(C_{g}-B_{g}\right) \cdot \omega_{y g} \cdot \omega_{z g} \\
& \sum M_{y g}=B_{g} \cdot \dot{\omega}_{y g}-\left(C_{g}-A_{g}\right) \cdot \omega_{x g} \cdot \omega_{z g}
\end{aligned}
$$

onde,

$A_{g}, B_{g}$ e $C_{g}$ são os momentos principais de inércia relativos aos eixos $x_{g}, y_{g} \mathrm{e} z_{g}$, respectivamente.

$$
\begin{aligned}
& \sum M_{x g}=T_{x r}-T_{x s}, \\
& \sum M_{y g}=T_{y r}-T_{y s},
\end{aligned}
$$

Os torques $T_{x s}$ e $T_{y r}$ são transmitidos através da torção das juntas. Considerando suas constantes de rigidez e de amortecimentos internos, temos:

$$
\begin{aligned}
& T_{x s}=k_{x} \cdot \theta_{x}+D_{x} \cdot \dot{\theta}_{x} \\
& T_{y r}=k_{y} \cdot \theta_{y}+D_{y} \cdot \dot{\theta}_{y}
\end{aligned}
$$

Substituindo e isolando-se os termos $T_{x r}$ e $T_{y s}$ vem:

$$
\begin{array}{r}
T_{x r}=k_{x} \cdot \theta_{x}+D_{x} \cdot \dot{\theta}_{x}+A_{g} \cdot \dot{\omega}_{x g}+\left(C_{g}-B_{g}\right) \cdot \omega_{y g} \cdot \omega_{z g} \\
T_{y s}=-k_{y} \cdot \theta_{y}-D_{y} \cdot \dot{\theta}_{y}-B_{g} \cdot \dot{\omega}_{y g}+\left(C_{g}-A_{g}\right) \cdot \omega_{x g} \cdot \omega_{z g}
\end{array}
$$

\subsection{Esforços no Rotor}

Antes de aplicarmos as equações de Euler ao rotor, descreveremos os momentos externos que estão sendo considerados. A junta elástica, que liga a cruzeta ao rotor (alinhado com o eixo $y_{r}$ ), é responsável pela transmissão de $T_{x r}$ e $T_{y r}$ à cruzeta. Um torque $T_{d}$, de arrasto, surge entre a carcaça e o rotor, proporcional à velocidade de rotação $N$, que atua na direção do eixo-motor $\left(z_{s}\right)$, e que produz as componentes $\left(-T d \cdot \theta_{y}\right)$ e $\left(T d \cdot \theta_{x}\right)$ nos eixos $x_{r}$ e $y_{r}$ respectivamente. Entre a carcaça e rotor também estão presentes momentos externos de amortecimento, $D r \cdot \dot{\theta}_{x c}$ e $D r \cdot \dot{\theta}_{y c}$, nas direções $x_{c}$ e $y_{c}$ da carcaça respectivamente, que são proporcionais às componentes da velocidade angular relativa entre ambos. Momentos externos aplicados pelas bobinas de torque, são usados para se fechar as malhas de controle $\left(M C_{x c}, M C_{y c}\right)$ também nas direções $x_{c}$ e $y_{c}$ da carcaça, respectivamente.

As equações para o rotor são dadas por:

$$
\begin{aligned}
& \sum M_{x r}=A \cdot \dot{\omega}_{x r}+(C-B) \cdot \omega_{y r} \cdot \omega_{z r} \\
& \sum M_{y r}=B \cdot \dot{\omega}_{y r}-(C-A) \cdot \omega_{x r} \cdot \omega_{z r}
\end{aligned}
$$

Onde,

$A, \quad B$ e $C$ são os momentos principais de inércia relativos aos eixos $x_{r}, y_{r}$ e $z_{r}$, respectivamente.

$$
\begin{aligned}
& \sum M_{x r}=M_{x}-T_{x r}-T_{d} \cdot \theta_{y} \\
& \sum M_{y r}=M_{y}-T_{y r}+T_{d} \cdot \theta_{x}
\end{aligned}
$$

e,

$M x=\left(M C_{x c}-D r \cdot \dot{\theta}_{x c}\right) \cos N t+\left(M C_{y c}-D r \cdot \dot{\theta}_{y c}\right) \operatorname{sen} N t$ 
$M y=-\left(M C_{x c}-D r \cdot \dot{\theta}_{x c}\right) \operatorname{sen} N t+\left(M C_{y c}-D r \cdot \dot{\theta}_{y c}\right) \cos N t$

As expressões (26) e (27) realizam a conversão de momentos expressos no referencial da carcaça para o referencial do rotor.

Aplicando as expressões de $T_{x r}$ e $T_{y r}$, temos:

$$
\begin{aligned}
& A \cdot \dot{\omega}_{x r}+(C-B) \cdot \omega_{y r} \cdot \omega_{z r}+k_{x} \cdot \theta_{x}+D_{x} \cdot \dot{\theta}_{x} \\
& +A_{g} \cdot \dot{\omega}_{x g}+\left(C_{g}-B_{g}\right) \cdot \omega_{y g} \cdot \omega_{z g}+T d \cdot \theta_{y}=M x \\
& B \cdot \dot{\omega}_{y r}-(C-A) \cdot \omega_{x r} \cdot \omega_{z r}+k_{y} \cdot \theta_{y}+D_{y} \cdot \dot{\theta}_{y} \\
& -T d \cdot \theta_{x}=M y
\end{aligned}
$$

Substituindo as expressões de $\omega_{x r}, \omega_{y r}, \omega_{z r}, \omega_{x g}, \omega_{y g}, \omega_{z g}$ (4-6 e 11-13), e suas derivadas , temos:

$\ddot{\theta}_{x}\left(A+A_{g}\right)+\dot{\theta}_{x} D_{x}+\theta_{x}\left[N^{2}\left(C-B+C_{g}-B_{g}\right)+k_{x}\right]+$

$\dot{\theta}_{y} N(C-A-B)+\theta_{y} T d=$

$-\dot{\omega}_{x s}\left(A+A_{g}\right)-\omega_{y s} N\left(C-B+C_{g}-B_{g}\right)+M x$

$\ddot{\theta}_{y} B+\dot{\theta}_{y} D_{y}+\theta_{y}\left[N^{2}(C-A)+k_{y}\right]-\dot{\theta}_{x} N(C-A-B)-\theta_{x} T d=$ $-\dot{\omega}_{y s} \cdot B+\omega_{x s} \cdot N \cdot(C-A)+M y$

Em seguida, substituindo as expressões de $\omega_{x s}, \omega_{y s}, \omega_{z s}(1-3)$, e suas derivadas em função das velocidades angulares da carcaça $\dot{\phi}_{x}, \dot{\phi}_{y}$ e reagrupando, tem-se:

$\ddot{\theta}_{x}\left(A+A_{g}\right)+\dot{\theta}_{x} D_{x}+\theta_{x}\left[N^{2}\left(C-B+C_{g}-B_{g}\right)+k_{x}\right]+$

$\dot{\theta}_{y} N(C-A-B)+\theta_{y} T d=$

$-\left(A+A_{g}\right)\left(\ddot{\phi}_{x} \cos N t+\ddot{\phi}_{y} \operatorname{sen} N t\right)$

$-N\left(C-B+A+C_{g}-B_{g}+A_{g}\right)\left(-\dot{\phi}_{x} \operatorname{sen} N t+\dot{\phi}_{y} \cos N t\right)+$

$M x$

$\ddot{\theta}_{y} B+\dot{\theta}_{y} D_{y}+\theta_{y}\left[N^{2} \cdot(C-A)+k_{y}\right]$

$-\dot{\theta}_{x} \cdot N \cdot(C-A-B)-\theta_{x} \cdot T d=$

$-B\left(-\ddot{\phi}_{x} \operatorname{sen} N t+\ddot{\phi}_{y} \cos N t\right)+$

$N(C-A+B)\left(\dot{\phi}_{x} \cos N t+\dot{\phi}_{y} \operatorname{sen} N t\right)+M y$

Interessa-nos a relação entre os movimentos de rotor e carcaça expressas no referencial da carcaça (que é o responsável pela medida fornecida pelo sensor). Assim sendo, devemos substituir as variáveis $\theta_{x}$ e $\theta_{y}$ pelas suas projeções no sistema da carcaça $\theta_{x c}$ e $\theta_{y c}$ usando as transformações expressas em (9) e (10). Tem-se então:

$\ddot{\theta}_{x c}\left\{\left(A+A_{g}\right) \cos N t\right\}+$

$\dot{\theta}_{x c}\left\{\begin{array}{l}-\left[2 N\left(A+A_{g}\right)+N(C-A-B)\right] \operatorname{sen} N t+ \\ \left(D_{x}+D_{r}\right) \cos N t\end{array}\right\}+$

$\theta_{x c}\left\{\begin{array}{l}-\left(T_{d}+N D_{x}\right) \operatorname{sen} N t \\ +\left[\begin{array}{c}N^{2} \cdot\left(C-B+C_{g}-B_{g}\right)+k_{x} \\ -N^{2} \cdot(C-A-B)-N^{2}\left(A+A_{g}\right)\end{array}\right] \cos N t\end{array}\right\}+$

$\ddot{\theta}_{y c}\left\{\left(A+A_{g}\right) \operatorname{sen} N t\right\}+$

$\dot{\theta}_{y c}\left\{\begin{array}{l}\left(D_{x}+D_{r}\right) \operatorname{sen} N t+ \\ {\left[N(C-A-B)+2 N\left(A+A_{g}\right)\right] \cos N t}\end{array}\right\}+$

$\left.\theta_{y c}\left\{\begin{array}{l}\left(N^{2} \cdot\left(C-B+C_{g}-B_{g}\right)+k_{x}\right) \\ -N^{2} \cdot(C-A-B)-N^{2}\left(A+A_{g}\right) \\ \left(T_{d}+N D_{x}\right) \cos N t\end{array}\right] \operatorname{sen} N t+\right\}=$

$-\left(A+A_{g}\right)\left(\ddot{\phi}_{x} \cos N t+\ddot{\phi}_{y} \operatorname{sen} N t\right)$

$-N\left(C-B+A+C_{g}-B_{g}+A_{g}\right)\left(-\dot{\phi}_{x} \operatorname{sen} N t+\dot{\phi}_{y} \cos N t\right)+$

$M_{x}$

$\ddot{\theta}_{x c}\{-B \operatorname{sen} N t\}+$

$\dot{\theta}_{x c}\left\{-\left(D_{y}+D_{r}\right) \operatorname{sen} N t-[2 B N+N(C-A-B)] \cos N t\right\}+$

$\theta_{x c}\left\{\left[\begin{array}{l}B N^{2}-N^{2}(C-A)+k_{y} \\ +N^{2} \cdot(C-A-B)\end{array}\right] \operatorname{sen} N t-\left(T_{d}-N D_{y}\right) \cos N t\right\}+$

$\ddot{\theta}_{y c}\{B \cos N t\}+$

$\dot{\theta}_{y c}\left\{-[2 N B+N \cdot(C-A-B)] \operatorname{sen} N t+\left(D_{y}+D_{r}\right) \cos N t\right\}+$

$\theta_{y c}\left\{\begin{array}{l}-\left(T_{d}+N D_{y}\right) \operatorname{sen} N t \\ +\left[N^{2}(C-A)+k_{y}-N^{2}(C-A-B)-N^{2} B\right] \cos N t\end{array}\right\}=$

$=-B \cdot\left(-\ddot{\phi}_{x} \cdot \operatorname{sen} N t+\ddot{\phi}_{y} \cdot \cos N t\right)$

$+N \cdot(C-A+B) \cdot\left(\dot{\phi}_{x} \cdot \cos N t+\dot{\phi}_{y} \cdot \operatorname{sen} N t\right)+M_{y}$

Admitindo juntas elásticas simétricas, tem-se:

$$
\begin{aligned}
& k_{x}=k_{y}=k \\
& D_{x}=D_{y}=D
\end{aligned}
$$


Além disso, para simplificar o conjunto de equações anteriores, executa-se as operações

$$
\begin{aligned}
& \text { “(34) } \cdot \cos N t-(35) \cdot \operatorname{sen} N t " \mathrm{e} \\
& \text { “(34) } \cdot \operatorname{sen} N t+(35) \cdot \cos N t "
\end{aligned}
$$

Utilizando também as definições (26) e (27), obtêm-se os resultados:

$$
\begin{aligned}
& \ddot{\theta}_{x c} I+\dot{\theta}_{x c}\left(D+D_{r}\right)+\theta_{x c}\left(k-N^{2} J\right)+\dot{\theta}_{y c} N\left(C+A_{g}\right)+ \\
& \theta_{y c}\left(T_{d}+N D\right)= \\
& -\ddot{\phi}_{x} I-\dot{\phi}_{y} N\left(C+A_{g}-J\right)-q_{x} \cos 2 N t-q_{y} \operatorname{sen} 2 N t+M C_{x} \\
& \ddot{\theta}_{y c} I+\dot{\theta}_{y c}\left(D+D_{r}\right)+\theta_{y c}\left(k-N^{2} J\right)+\dot{\theta}_{x c} N\left(-C-A_{g}\right) \\
& -\theta_{x c}\left(T_{D}+D N\right)= \\
& -\ddot{\phi}_{y} I+\dot{\phi}_{x} N\left(C+A_{g}-J\right)-q_{x} \operatorname{sen} 2 N t+q_{y} \cos 2 N t+M C_{y}(37)
\end{aligned}
$$

onde,

$$
\begin{aligned}
& I=\frac{1}{2}\left(A+B+A_{g}\right), \\
& J=\frac{1}{2}\left(A_{g}+B_{g}-C_{g}\right), \\
& q_{x}=\ddot{\theta}_{x c} \Delta I+\theta_{x c} N^{2} \Delta J+\dot{\theta}_{y c} 2 N \Delta I+\ddot{\phi}_{x} \Delta I+\dot{\phi}_{y} N\left(\Delta I+\Delta I_{S}\right) \\
& q_{y}=\ddot{\theta}_{y c} \Delta I+\theta_{y c} N^{2} \Delta J-\dot{\theta}_{x c} 2 N \Delta I+\ddot{\phi}_{y} \Delta I-\dot{\phi}_{x} N\left(\Delta I+\Delta I_{S}\right) \\
& \Delta I=\frac{1}{2}\left(A-B+A_{g}\right) \\
& \Delta I_{S}=\frac{1}{2}\left(A-B+C_{g}-B_{g}\right) \\
& \Delta J=\frac{1}{2}\left(C_{g}-A_{g}-B_{g}\right)
\end{aligned}
$$

Chegamos assim, aos resultados (36) e (37), que, a menos dos termos relativos ao movimento da carcaça, correspondem às expressões apresentadas no guia de especificação de testes e procedimentos da IEEE (1989) para o caso de cruzeta e rotor com simetria axial. Nota-se os esforços de reação relacionados com as inércias da cruzeta, que oscilam com o dobro da freqüência de rotação do rotor. Com relação a este resultado, é interessante observar que as restrições cinemáticas da ligação entre rotor e cruzeta impõem uma oscilação na cruzeta com freqüência " $2 \mathrm{~N}$ " quando o rotor assume uma orientação fixa no espaço. As oscilações em " $2 \mathrm{~N}$ " presentes nos sinais originados do sensor costumam ser atenuadas, nos circuitos de condicionamento, por filtros de rejeição de banda.

\section{ANÁLISE DAS RESPOSTAS EM MALHA ABERTA}

\subsection{Condição Ideal}

Vamos considerar um giroscópio perfeitamente simétrico $\left(A=B, A_{g}=B_{g}\right)$ e com inércias da cruzeta muito menores do que as inércias do rotor $\left(A_{g}, C_{g}<<A, C\right)$. Neste caso, podemos desprezar os coeficientes presentes em $q_{x}$ e $q_{y \text {., }}$, além das inércias da cruzeta nas equações anteriores. Sua resposta em malha aberta $\left(M C_{x}=M C_{y}=0\right)$, é dada por:

$$
\begin{aligned}
& \ddot{\theta}_{x c} I+\dot{\theta}_{x c}\left(D+D_{r}\right)+\theta_{x c}\left(k-N^{2} J\right)+\dot{\theta}_{y c} N C \\
& +\theta_{y c}\left(T_{d}+N D\right)=-\ddot{\phi}_{x} I-\dot{\phi}_{y} N C \\
& \ddot{\theta}_{y c} I+\dot{\theta}_{y c}\left(D+D_{r}\right)+\theta_{y c}\left(k-N^{2} J\right)-\dot{\theta}_{x c} N C \\
& -\theta_{x c}\left(T_{d}+N D\right)=-\ddot{\phi}_{y} I+\dot{\phi}_{x} N C
\end{aligned}
$$

A condição de sintonia, ou seja, para $N=N_{0}=\sqrt{k / J}$, representa a situação em que o momento aplicado pela torção das juntas elásticas é contrabalançado pela reação da cruzeta (torque devido à sua aceleração e binário giroscópico) ao movimento que lhe é imposto. Neste caso, sem esforços de amortecimento, o rotor giraria livre no espaço , como é de se esperar de um sensor giroscópico ideal. Ou seja, supondo carcaça e rotor inicialmente alinhados, as equações acima forneceriam:

$$
\begin{aligned}
& \theta_{x c}(t)=-\phi_{x}(t) \\
& \theta_{y c}(t)=-\phi_{y}(t)
\end{aligned}
$$

\subsection{Funções de Transferência em Malha Aberta na Condição de Sintonia}

A constante de tempo de um DTG é um fator de importância na determinação da qualidade do mesmo. Ela está relacionada aos esforços de amortecimento que atuam sobre o rotor. Ao girarmos a carcaça, mesmo sem a ação das bobinas de torque, estes esforços implicariam no alinhamento da carcaça e rotor através do movimento de precessão do último. Quanto maior este tempo de alinhamento, ou seja, maior a constante de tempo, melhor a qualidade do sensor. Outro fenômeno que pode prejudicar o desempenho do giroscópio é a nutação do rotor, que ocasiona oscilações na leitura do sensor. Como em Craig (1972 b), as funções de transferência, obtidas a partir das equações anteriores, podem ser analisadas à luz desses fenômenos, conforme será mostrado a seguir.

Aplicando a transformada de Laplace a (38) e (39) vem: 


$$
P_{1}(s) \cdot \theta_{x c}(s)+P_{2}(s) \cdot \theta_{y c}(s)=-P_{3}(s) \cdot \phi_{x}(s)-P_{4}(s) \cdot \phi_{y}(s)
$$

$-P_{2}(s) \cdot \theta_{x c}(s)+P_{1}(s) \cdot \theta_{y c}(s)=P_{4}(s) \cdot \phi_{x}(s)-P_{3}(s) \cdot \phi_{y}(s)$

Os polinômios em $s$ são definidos por:

$$
\begin{aligned}
& P_{1}(s)=I \cdot s^{2}+\left(D+D_{r}\right) \cdot s=I s\left[s+\frac{\left(D+D_{r}\right)}{I}\right]=I s\left(s+\frac{1}{\tau_{1}}\right) \\
& P_{2}(s)=N \cdot\left(C+A_{g}\right) \cdot s+\left(T_{d}+N D\right)=I \omega_{n}\left(s+\frac{1}{\tau}\right) \\
& P_{3}(s)=I s^{2} \\
& P_{4}(s)=N\left(C+A_{g}-J\right) \cdot s= \\
& I \frac{N\left(C+A_{g}\right)}{I} s-\frac{\left(A_{g}+B_{g}-C_{g}\right) N}{2} s=I \omega_{n} s\left(1-\frac{1}{2 F m}\right)
\end{aligned}
$$

onde,

$$
\omega_{n}=\frac{\left(C+A_{g}\right)}{I} N
$$

é a freqüência de nutação, e

$$
\begin{aligned}
& \frac{1}{\tau_{1}}=\frac{D+D r}{I} \\
& \frac{1}{\tau}=\left(\frac{D}{I}+\frac{D_{d}}{I}\right) \frac{N}{\omega_{n}} 1 \\
& D_{d}=T_{d} / N \\
& F m=\frac{\left(C+A_{g}\right)}{\left(A_{g}+B_{g}-C_{g}\right)}
\end{aligned}
$$

A matriz de transferência, relacionando os movimentos do rotor e carcaça é dada por:

$$
\left[\begin{array}{l}
\theta_{x c}(s) \\
\theta_{y c}(s)
\end{array}\right]=\left[\begin{array}{ll}
G_{11}(s) & G_{12}(s) \\
G_{21}(s) & G_{22}(s)
\end{array}\right] \cdot\left[\begin{array}{l}
\phi_{x}(s) \\
\phi_{y}(s)
\end{array}\right]
$$

onde,

$$
\begin{aligned}
& G_{11}(s)=G_{22}(s)=-\frac{P_{1} P_{3}+P_{2} P_{4}}{P_{1}^{2}+P_{2}^{2}} \\
& G_{12}(s)=-G_{21}(s)=\frac{-P_{1} P_{4}+P_{2} P_{3}}{P_{1}^{2}+P_{2}^{2}}
\end{aligned}
$$

Estas funções de transferência acima serão expressas em função das dinâmicas de precessão e nutação do rotor. Inicialmente, tem-se:

\footnotetext{
${ }^{1}$ Nota-se uma diferença em relação à expressão 18 de Craig (1972b), pois esta não apresenta os parênteses, provavelmente devido a um erro de impressão.
}

$$
P_{1}^{2}(s)+P_{2}^{2}(s)=I^{2}\left[s^{2}\left(s+\frac{1}{\tau_{1}}\right)^{2}+\omega_{n}^{2}\left(s+\frac{1}{\tau}\right)^{2}\right]
$$$$
P_{1}(s) P_{3}(s)+P_{2}(s) P_{4}(s)=
$$$$
=I^{2} s\left\{s^{2}\left[\left(s+\frac{1}{\tau}\right)+\left(\frac{1}{\tau_{1}}-\frac{1}{\tau}\right)\right]+\omega_{n}^{2}\left(1-\frac{1}{2 F m}\right)\left(s+\frac{1}{\tau}\right)\right\}
$$$$
=I^{2} s\left\{\left(s+\frac{1}{\tau}\right)\left[s^{2}+\omega_{n}^{2}\left(1-\frac{1}{2 F m}\right)\right]+\left(\frac{1}{\tau_{1}}-\frac{1}{\tau}\right) s^{2}\right\}
$$

$$
\begin{aligned}
P_{2}(s) P_{3}(s)-P_{1}(s) P_{4}(s) & =I^{2} \omega_{n} s^{2}\left[\frac{1}{2 F m} s+\left(\frac{1}{\tau}-\frac{1}{\tau_{1}}\right)+\frac{1}{2 \tau_{1} F m}\right] \\
& \cong I^{2} \omega_{n} s^{2}\left[\frac{1}{2 F m} s+\left(\frac{1}{\tau}-\frac{1}{\tau_{1}}\right)\right]
\end{aligned}
$$

A fatoração do denominador das funções de transferência pode ser imposta em termos das dinâmicas de precessão e nutação do rotor como segue:

$$
\begin{aligned}
& P_{1}^{2}(s)+P_{2}^{2}(s)=I^{2}\left[s^{2}\left(s+\frac{1}{\tau_{1}}\right)^{2}+\omega_{n}^{2}\left(s+\frac{1}{\tau}\right)^{2}\right] \cong \\
& \cong I^{2}\left(s+\frac{1}{\tau}\right)^{2}\left(s^{2}+\frac{2}{\tau_{n}} s+\omega_{n}^{2}\right)
\end{aligned}
$$

onde $\tau_{n}$ é a constante de tempo da dinâmica de nutação.

Utiliza-se a igualdade dos polinômios acima, pois os demais coeficientes relativos à potência $s^{2}$ são desprezados face a $\omega_{n}^{2}$, bem como é desprezado $1 /\left(\tau \cdot \tau_{n}\right)$ no termo referente a $s$. A constante de tempo de nutação é determinada a partir da igualdade dos termos em $s^{3}$ :

$$
\frac{1}{\tau_{n}}=\left(\frac{1}{\tau_{1}}-\frac{1}{\tau}\right)=\frac{D_{r}}{I}+\frac{D}{I}\left(1-\frac{N}{\omega_{n}}\right)-\frac{D_{d}}{I} \frac{N}{\omega_{n}}
$$

O significado físico dos termos desta expressão é discutido na seção 4.4 .

A influência do termo $1 /\left(\tau \cdot \tau_{n}\right)$ é também desprezada para se chegar à aproximação do numerador de $G_{11}$ :

$$
\begin{aligned}
& P_{1}(s) P_{3}(s)+P_{2}(s) P_{4}(s)= \\
& =I^{2} s\left\{\left(s+\frac{1}{\tau}\right)\left[s^{2}+\omega_{n}^{2}\left(1-\frac{1}{2 F m}\right)\right]+\left(\frac{1}{\tau_{1}}-\frac{1}{\tau}\right) s^{2}\right\}= \\
& =I^{2} s\left\{\left(s+\frac{1}{\tau}\right)\left[s^{2}+\frac{1}{\tau_{n}} s+\omega_{n}^{2}\left(1-\frac{1}{2 F m}\right)\right]\right\}
\end{aligned}
$$


Admitindo-se o cancelamento de pólos e zeros relativos à dinâmica de nutação para o canal direto, tem-se:

$$
G_{11}(s)=-\frac{s}{\left(s+\frac{1}{\tau}\right)}
$$

A função de transferência relativa ao canal cruzado é dada por:

$$
\begin{aligned}
& G_{12}(s)=\frac{s^{2}\left[\left(\omega_{n} / 2 F m\right) s-\left(\omega_{n} / \tau_{n}\right)\right]}{\left(s+\frac{1}{\tau}\right)^{2}\left(s^{2}+\frac{2}{\tau_{n}} s+\omega_{n}{ }^{2}\right)} \cong \\
& \cong s^{2}\left[\left(1 / 2 F m \omega_{n}\right) s-\left(1 / \omega_{n} \tau_{n}\right)\right]\left[\frac{1}{\left(s+\frac{1}{\tau}\right)^{2}}-\frac{1}{\left(s^{2}+\frac{2}{\tau_{n}} s+\omega_{n}{ }^{2}\right)}\right]
\end{aligned}
$$

Dada a magnitude de $G_{12}(s)$, pode-se considerar somente a contribuição de $G_{11}(s)$ na resposta do rotor a um pequeno deslocamento angular na carcaça. Ou seja, para as entradas em degrau com amplitudes $\phi_{x}(0)$ e $\phi_{y}(0)$, tem-se:

$$
\begin{aligned}
& \theta_{x c}(t)=-\phi_{x}(0) e^{-t / \tau} \\
& \theta_{y c}(t)=-\phi_{y}(0) e^{-t / \tau}
\end{aligned}
$$

As expressões anteriores mostram que o alinhamento entre rotor e carcaça se dá num intervalo de tempo determinado pela constante de tempo de precessão do giroscópio, e que este será tanto mais lento (o que é desejável para aproximar o desempenho do sensor ao caso ideal) quanto menores forem os amortecimentos envolvidos.

\subsection{Funções de Transferência em Malha Aberta na Condição fora de Sintonia}

Na prática, o DTG opera bem próximo à condição ideal de sintonia, devido a pequenas imprecisões no projeto e na fabricação, e pela variação de seus parâmetros com o tempo. Uma característica importante para resguardar o bom desempenho nesse caso é a relação entre inércias do rotor e cruzeta. Esta é expressa pela chamada "figura de mérito", Fm, do giroscópio. Sua influência será mostrada seguir.

Para pequenos desvios em relação à condição de sintonia, tem-se:

$$
\begin{aligned}
& \left(k-N^{2} J\right)=J\left(N_{0}^{2}-N^{2}\right)= \\
& =J\left[(N+\delta N)^{2}-N^{2}\right]=J\left(\delta N^{2}+2 n \delta N\right)
\end{aligned}
$$

Para $\delta N<<$, pode-se fazer a aproximação:

$$
\begin{aligned}
& \left(k-N^{2} J\right) \cong 2 N \delta N= \\
& =I \frac{\left(C+A_{g}\right)}{I} N \frac{\delta N}{\left(C+A_{g}\right) / 2 J}=I \omega_{n} \frac{\delta N}{F m}
\end{aligned}
$$

Neste caso, a expressão do polinômio $P_{1}(s)$ e o numerador de $G_{12}(s)$ são modificados para:

$$
\begin{aligned}
& P_{1}(s)=I\left[s^{2}+\left(\frac{1}{\tau}+\frac{1}{\tau_{n}}\right) s+\omega_{n} \frac{\delta N}{F m}\right] \\
& P_{2}(s) P_{3}(s)-P_{1}(s) P_{4}(s)= \\
= & I^{2} \omega_{n} s\left\{s\left(s+\frac{1}{\tau}\right)-\left[s^{2}+\left(\frac{1}{\tau}+\frac{1}{\tau_{n}}\right) s+\omega_{n} \frac{\delta N}{F m}\right]\left(1-\frac{1}{2 F m}\right)\right\} \\
= & I^{2} \omega_{n} s\left\{\frac{1}{2 F m} s^{2}+\frac{1}{2 F m}\left(\frac{1}{\tau}+\frac{1-2 F m}{\tau_{n}}\right) s+\omega_{n} \frac{\delta N}{F m}\left(1-\frac{1}{2 F m}\right)\right\} \\
\cong & I^{2} \omega_{n} s\left\{\frac{1}{2 F m} s^{2}-\frac{1}{\tau_{n}} s-\omega_{n} \frac{\delta N}{F m}\right\}
\end{aligned}
$$

Sabendo-se que $\frac{\delta N}{F m}<<\omega_{n}$, pode-se considerar a expressão do numerador de $G_{l l}$ inalterada em relação a (48), e a expressão do denominador das funções de transferência pode ser aproximada para:

$$
P_{1}^{2}(s)+P_{2}^{2}(s)=I^{2}\left[\left(s+\frac{1}{\tau}\right)^{2}+\left(\frac{\delta N}{F m}\right)^{2}\right]\left(s^{2}+\frac{2}{\tau_{n}} s+\omega_{n}^{2}\right)
$$

Portanto, as novas expressões das funções de transferência passam a ser:

$$
\begin{gathered}
G_{11}(s)=-\frac{s\left(s+\frac{1}{\tau}\right)\left[s^{2}+\frac{1}{\tau_{n}} s+\omega_{n}^{2}\left(1-\frac{1}{2 F m}\right)\right]}{\left[\left(s+\frac{1}{\tau}\right)^{2}+\left(\frac{\delta N}{F m}\right)^{2}\right]\left(s^{2}+\frac{2}{\tau_{n}} s+\omega_{n}^{2}\right)} \\
G_{12}(s)=\frac{\left.\omega_{n} s \cdot\left[\frac{1}{2 F m} s^{2}-\frac{1}{\tau_{n}} s-\omega_{n} \frac{\delta N}{F m}\right)\right]}{\left[\left(s+\frac{1}{\tau}\right)^{2}+\left(\frac{\delta N}{F m}\right)^{2}\right]\left(s^{2}+\frac{2}{\tau_{n}} s+\omega_{n}{ }^{2}\right)}
\end{gathered}
$$

A Fig. 7 mostra a resposta em freqüência de um DTG, de acordo com a norma apresentada pelo IEEE (1989). Esta é a forma de resultado produzida a partir das expressões (56) e (57), utilizando valores com a mesma ordem de grandeza dos apresentados na tabela 1 . 


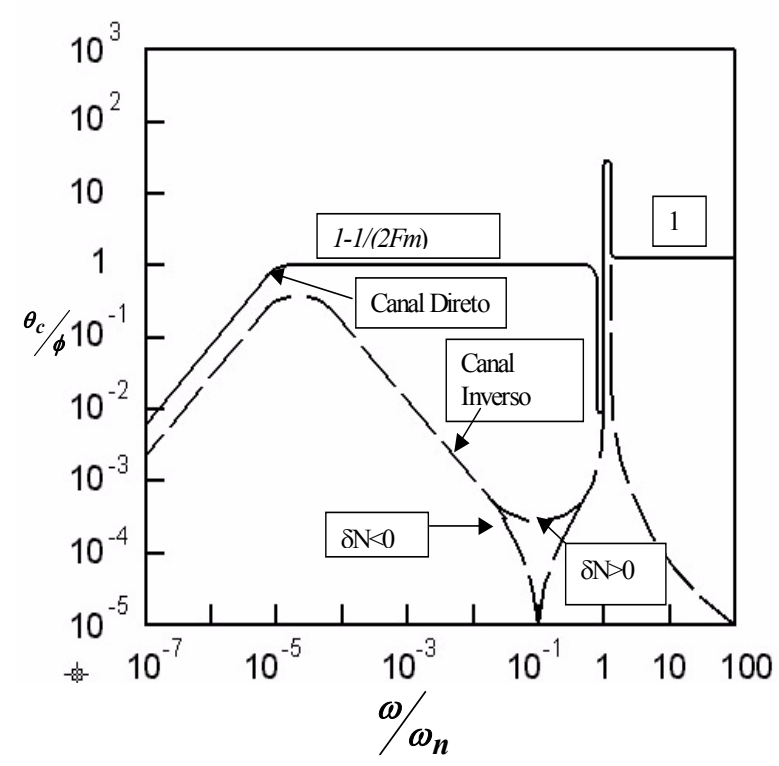

Figura 7 - Resposta em Freqüência para um DTG típico em malha aberta (reproduzido a partir de IEEE, 1989).

Como na seção anterior, após a decomposição em frações parciais da expressão de $G_{12}(s)$, para o cálculo da resposta em degrau do sistema (entradas iguais a $\left.\frac{\phi_{x}(0)}{s} \mathrm{e} \frac{\phi_{y}(0)}{s}\right)$, despreza-se fatores de menor amplitude nas freqüências de nutação e $\frac{\delta N}{F m}$. Além disso, supõe-se também o cancelamento de pólos e zeros correspondentes à dinâmica de nutação. Têm-se, dessa forma, os resultados:

$$
\begin{gathered}
G_{11}(s)=-\frac{s\left(s+\frac{1}{\tau}\right)}{\left[\left(s+\frac{1}{\tau}\right)^{2}+\left(\frac{\delta N}{F m}\right)^{2}\right]} \\
G_{12}(s)=\frac{-s(\delta N / F m)}{\left[\left(s+\frac{1}{\tau}\right)^{2}+\left(\frac{\delta N}{F m}\right)^{2}\right]}
\end{gathered}
$$

Sendo assim, a resposta em degrau do movimento do rotor é dada por:

$$
\theta_{x c}(t)=-\phi_{x}(0) e^{-t / \tau} \cos \left(\frac{\delta N}{F m}\right) t-\phi_{y}(0) e^{-t / \tau} \operatorname{sen}\left(\frac{\delta N}{F m}\right) t
$$

\footnotetext{
${ }^{2}$ Note a diferença de sinal no segundo termo do lado direito da equação em relação à eq. 25 de Craig (1972 b)
}

$$
\theta_{y c}(t)=\phi_{x}(0) e^{-t / \tau} \operatorname{sen}\left(\frac{\delta N}{F m}\right) t-\phi_{y}(0) e^{-t / \tau} \cos \left(\frac{\delta N}{F m}\right) t
$$

Observa-se então a importância de se utilizar altas magnitudes dos valores da figura de mérito para atenuar os efeitos de desvios na sintonia do DTG, que, em conjunto com baixos valores de amortecimento, deixa seu comportamento próximo ao de um giroscópio ideal.

\subsection{Influência dos Parâmetros de Amortecimento}

É interessante observar o papel de cada tipo de amortecimento nas dinâmicas associadas com a precessão e a nutação do rotor. Nota-se, de acordo com as expressões (60) e (61), o papel da constante de tempo do movimento de precessão, o qual traz lentamente o rotor a um alinhamento com a carcaça. Os amortecimentos internos das juntas elásticas são responsáveis pela perda de energia do rotor, quando este assume uma atitude diferente da carcaça, devido ao movimento oscilatório assumido pela cruzeta. Também presente na constante de tempo de precessão, o momento de amortecimento " $T_{d}$ ", proveniente do meio gasoso entre rotor e carcaça. $O$ momento, que atua na direção do eixo de "spin" do rotor é praticamente igual a " $T_{d}$ ", e uma componente perpendicular ao eixo do rotor, “ $T_{d} \theta$ ”, resulta da composição entre o momento motc 0 , conforme ilustra a Fig. 8.

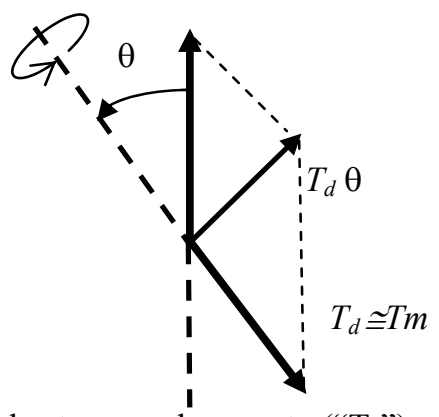

Figura 8 -Atuação dos torques de arrasto (" $\mathrm{T}_{\mathrm{d}}$ ") e motor sobre o rotor .

Este momento resultante, pelo efeito giroscópico, induz no rotor uma rotação do seu eixo de "spin" em torno de um eixo perpendicular ao plano da figura, no sentido de aproxima-lo ao eixo motor.

No caso da nutação, este movimento oscilatório seria auto-sustentável, se não houvesse as perdas por amortecimento. Ele representa a troca de energia entre um grau de liberdade e outro. Como é de se esperar, o amortecimento para um movimento oscilatório entre

\footnotetext{
${ }^{3}$ Note a diferença de sinal no primeiro termo do lado direito da equação em relação à eq. 26 de Craig (1972 b)
} 
carcaça e rotor possui um papel dissipativo, bem como o amortecimento interno das juntas elásticas para valores típicos da velocidade de nutação $\left(\omega_{n} \cong 1,7 N\right)$, o que está representado na expressão (47). $O$ caso de freqüência de rotação motora e de nutação coincidentes corresponde ao caso de pouca utilidade em que o rotor é esférico, e implica na anulação entre si dos valores das variáveis de movimento que multiplicam " $D$ " em (36) e (37). Finalmente, o sinal negativo do último termo em (47) representa o papel atenuador do amortecimento da nutação originado da ação da componente " $T_{d} \theta$ ", já que esta parcela induz um movimento de rotação do eixo do rotor, conforme explicado no parágrafo precedente.

Ao contrário da precessão, o amortecimento produz um efeito desejável ao atenuar o fenômeno de nutação. Mesmo assim, neste caso também utiliza-se comumente um filtro de rejeição de banda, com o papel de eliminar o sinal espúrio da nutação presente na leitura do sensor.

\section{CONCLUSÕES}

Neste trabalho, as funções de transferência, relacionando movimentos de carcaça e rotor, foram derivadas a partir de simplificações aplicadas ao conjunto completo de equações de movimento de um giroscópio sintonizado dinamicamente. A abordagem utilizada visou o esclarecimento e integração de resultados comuns na literatura.

Próximos passos neste estudo incluem o tratamento do modelo de erros do sensor e sua compensação a partir de resultados de ensaios, para a integração do mesmo num algoritmo de navegação. Pretende-se também investigar, à luz destes resultados o papel de algumas estratégias de controle do movimento do rotor.

A importância do desenvolvimento autônomo de sistemas inerciais, voltados a diversas aplicações de interesse no país, sugere o estabelecimento de uma massa crítica de pesquisadores dedicados ao seu estudo. A divulgação da teoria do "DTG" representa uma contribuição neste sentido, focalizando um sensor com potencialidade já comprovada pelo desenvolvimento de protótipos no Centro Tecnológico da Marinha em São Paulo.

\section{AGRADECIMENTOS}

Os autores agradecem o apoio financeiro da Agência Espacial Brasileira através do programa "UNIESPAÇO”.

\section{REFERÊNCIAS BIBLIOGRÁFICAS}

Craig, R. J. G. Theory of Operation of an Elastically Supported, Tuned Gyroscope, IEEE Trans. on
Aerospace and Electronic Systems, p 280-288. vol AES-8, No 3, maio. 1972a.

Craig, R. J. G. Theory of Errors of a Multigimbal, Elastically Supported, Tuned Gyroscope, IEEE Trans. on Aerospace and Electronic Systems, p 289-297. vol AES-8, No 3, maio. 1972b.

Garrotti, J.C. Modelagem e Simulação de um Girômetro Sintonizado Dinamicamente em um CAD Eletrônico. Dissertação de Mestrado. Instituto de Pesquisas Espaciais. INPE 9702-TDI/856. 2003.

IEEE - Std 813-1988. Specification Format Guide and Test Procedure for Two-Degree-of Freedom, Dynamically Tuned Gyros. IEEE, 1989, New York. USA.

Junqueira, F. C. Desenvolvimento de um Giroscópio Sintonizado Dinamicamente- DTG. Dissertação de Mestrado. Escola Politécnica da USP. 2003.

Junqueira, F. C., E. A. de Barros - Development of a Dynamically Tuned Gyroscope- DTG. Proc. Of Cobem , 2003.

Lawrence, A. Modern Inertial Technology. Springer Verlag, $2^{\text {nd }}$ Ed., 1992. 